\title{
Global Perspectives in Pharmacovigilance
}

\section{Fabrizio De Ponti*}

Department of Medical and Surgical Sciences, University of Bologna, Bologna, Italy

The inaugural issue of the journal is a good opportunity to highlight the changing attitute of stakeholders in the pharmaceutical field towards pharmacovigilance. Indeed, not much time has elapsed since the early age, when drug safety matters used to be conducted in secret to avoid alarming the public. Today, the need for transparency has dramatically changed the picture and pharmacovigilance has become a multifaceted field. The World Health Organization defines pharmacovigilance as the science and activities relating to the detection, assessment, understanding and prevention of adverse effects or any other drugrelated problem [1].

Safety issues need to be carefully addressed in a coherent way, because information is gathered from different sources (pre-marketing trials, post-approval safety studies, spontaneous reports, etc.) and from different parts of the world (e.g. FDA-AERS, Vigibase, Eudravigilance) according to different standards. Consistency in collection of safety data is mandatory to reduce bias and facilitate analysis/interpretation in a global perspective.

Six issues deserve to be mentioned here because they can also provide a clearer picture of the goals of the journal. The first issue is that the goal of pharmacovigilance is not only to prevent harm by identifying safety problems, but also to promote appropriate use of medicines by providing timely information on safety aspects and promoting a more balanced risk/benefit assessment by the prescriber.

A second issue is that there should be agreement on the definition of adverse reactions. Notably, the European Medicines Agency now considers that adverse reactions may arise from use of the product both within and outside the terms of the marketing authorization. Conditions of use outside the marketing authorization include offlabel use, overdose, misuse, abuse and medication errors [2]. Including off-label use clearly recognizes that a different indication/dose/route of administration of a medication may imply a different risk/benefit balance and calls for specific attention [3].

Third aspect to be addressed is that some experts still consider pharmacovigilance at the signal detection level an art as much as a0 science [4]. On the other hand, after a hypothesis for a signal is generated, rigorous scientific methods play a key role in safeguarding individual and public health. In my experience, for instance, when data mining techniques of spontaneous reports find a disproportionality signal for a drug, rigorous assessment is essential to avoid two possible scenarios: a) overlooking a true safety problem or b) emphasizing the importance of a biased signal [5]. In the latter scenario, withdrawing a drug would be a very crude tool to prevent harm. One goal of pharmacovigilance should be to prevent harm by identifying patients at risk and maintain a useful drug for the rest.

Fourth aspect that started to get appreciations in recent years is the pharmacoepidemiological perspective: once a safety issue has been identified, it is important to consider drug utilization data to assess risk in a public health perspective. This is particularly so for very rare adverse events, such as Torsades de Pointes, which may pose significant public health problems especially when exposure of the population is significant $[6,7]$.
Fifth aspect is that, until recently a significant difference existed between US and European pharmacovigilance databases of spontaneous reports: involvement of citizens was minimal in Europe. The new European Directive now places emphasis on reporting suspected adverse reactions by both healthcare professionals and patients. Because of the different perception of adverse reactions by doctors and patients, this will pose new research questions as well new challenges when analyzing spontaneous reports.

Sixth and final issue, which is so important for an open access Journal, is transparency, whose importance is increasingly perceived by regulatory agencies such as the Food and Drug Administration (the freely available version of the FDA AERS database represents an important source to detect signals) and the European Medicines Agency, which has also started to provide free (although still limited) access to data from spontaneous reports.

In this multifaceted field, how can the Journal of Pharmacovigilance serve the scientific community and promote better use of medicinal products? All the items mentioned above offer ideas for future submissions. The journal will publish quality case reports, original research and review articles about all safety aspects of medications. Even when adverse events are common, the lack of reliable quantitative information does not allow accurate assessment of the risk/benefits profile. Especially critical evaluation and validation of signals generated in pharmacovigilance still relies too much on expert's view rather than on standardized and shared methods. In this area, the journal can certainly foster discussion and fill the gap.

In addition, the full open-access policy makes all published material immediately and freely available and is particularly relevant when it comes to information about drugs safety, all the more so in the perspective of increased transparency promoted by health authorities and regulatory agencies. In today's wired world, the Open Access publication policy of the Journal has the potential to reach a far larger audience than any subscription-based journal. We do hope that also for investigators and readers this Journal can provide a scientific forum for discussion and will welcome addition to the existing literature.

\section{References}

1. World Health Organization (2006) The safety of medicines in public health programmes: pharmacovigilance an essential tool.

2. European Medicines Agency (2012) Guideline on good pharmacovigilance practices (GVP)

*Corresponding author: Fabrizio De Ponti, Pharmacology Unit, Department of Medical and Surgical Sciences, University of Bologna, Via Irnerio, 4840126 Bologna BO, Italy, E-mail: fabrizio.deponti@unibo.it

Received January 19, 2013; Accepted January 21, 2013; Published January 24 2013

Citation: De Ponti F (2013) Global Perspectives in Pharmacovigilance. J Pharmacovigilance 1: e108. doi:10.4172/2329-6887.1000e108

Copyright: () 2013 De Ponti F. This is an open-access article distributed under the terms of the Creative Commons Attribution License, which permits unrestricted use, distribution, and reproduction in any medium, provided the original author and source are credited. 
3. Edwards IR (2011) Off-label pharmacovigilance. Drug Saf 34: 795-797.

4. Edwards IR (2012) Good pharmacovigilance practice and the curate's egg Drug Saf 35: 429-435.

5. Raschi E, Piccinni C, Poluzzi E, Marchesini G, De Ponti F (2011) The association of pancreatitis with antidiabetic drug use: gaining insight through the FDA pharmacovigilance database. Acta Diabetol.
6. Poluzzi E, Raschi E, Motola D, Moretti U, De Ponti F (2010) Antimicrobials and the risk of torsades de pointes: the contribution from data mining of the US FDA Adverse Event Reporting System. Drug Saf 33: 303-314.

7. Raschi E, Poluzzi E, Zuliani C, Muller A, Goossens H, et al. (2009) Exposure to antibacterial agents with QT liability in 14 European countries: trends over an 8-year period. Br J Clin Pharmacol 67: 88-98. 